\title{
Commentary: Shouldering ever heavier burdens: Aortic root replacement in the context of depressed left ventricular ejection fraction
}

\author{
James M. Meza, MD, MSc
}

From the Department of Surgery, Duke University Medical Center, Durham, NC.

Disclosure: Author has nothing to disclose with regard to commercial support.

Received for publication Nov 11, 2018; accepted for publication Nov 12, 2018; available ahead of print Dec 28, 2018

Address for reprints: James M. Meza, MD, MSc, 2301 Erwin Rd, Box 3443 n, Durham, NC 27705 (E-mail: james. meza@duke.edu).

J Thorac Cardiovasc Surg 2019;158:994-5

0022-5223/\$36.00

Copyright (c) 2018 by The American Association for Thoracic Surgery

https://doi.org/10.1016/j.jtcvs.2018.11.043

Heart failure contributes to 1 in 9 deaths in the United States and has long been associated with poor outcomes after cardiac surgery. ${ }^{1,2}$ Langer and colleagues ${ }^{3}$ here present an important study regarding the effect of decreased ejection fraction (EF) on outcomes after aortic root replacement (ARR). They performed an observational cohort study of 595 patients who underwent ARR at their institution over 8 years, of which a reduced EF was present in 110 $(18 \%)$. The authors rigorously investigated the impact of decreased EF on midterm outcomes using restricted cubic splines and time-to-event analyses.

This study represents the first dedicated to understanding the role of decreased EF on outcomes after ARR and has 3 primary findings. First, the authors demonstrate that EF is an independent risk factor for mortality after ARR and is nearly linearly related to adjusted mortality. Notably, when $\mathrm{EF}=25 \%$, the risk of death is double the risk when $\mathrm{EF}=50 \%$. In addition, because this study included patients with seemingly disparate indications for ARR, including type A aortic dissection, thoracic aortic aneurysm, and infective endocarditis, their data also clarify that the indication for surgery does not overwhelm the specific effect of EF on mortality in patients undergoing ARR. The subgroup analysis, including only those patients undergoing ARR for thoracic aortic aneurysms, demonstrated similar patterns of increasing morbidity and mortality with decreasing EF. Finally, the authors conclude that in the elective setting, the recommendation for ARR should be individualized to each patient. They astutely do not attempt to suggest a revision of the current sized-based guidelines for ARR or the establishment of a blunt cutoff EF value. Instead, they advocate that the decision to operate be made within the context of the patient's degree of compromised EF and expected postoperative morbidity and mortality versus the specific risk of an aortic complication.

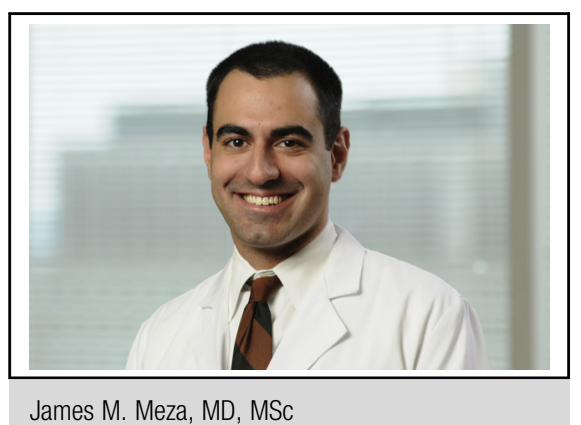

James M. Meza, MD, MSc

Central Message

Given the independent association of depressed ejection fraction with morbidity and mortality after aortic root replacement, individualized preoperative counseling that considers a patient's risk of aortic events is crucial.

See Article page 984.
This analysis is primarily limited by a lack of granularity around the specific etiologies of decreased EF in the study population. Detailed echocardiographic data describing aortic valve function, competency, and root anatomy were not included. Therefore, the impact of the etiology on postoperative prognosis and its potential use in preoperative counseling remains unexplored and represents an important topic for future analysis.

In the current era, the comorbidity burden for each patient continues to become heavier. As demonstrated here, impressively low rates of morbidity and mortality after this technically complex operation in high-risk patients have been achieved, often by using the expanding armamentarium of medical optimization and supportive modalities including mechanical circulatory support. In contrast, the availability of these advanced methods does not eliminate the need for frank, face-to-face counseling of the individual patient by a surgeon. Langer and colleagues now empower the surgeon to provide data-driven guidance to the patient with a low EF for whom ARR is indicated. The association of low EF with mortality can be integrated into detailed preoperative discussions of the individual patient's concern for future aortic events and willingness to accept the possibility of life-altering risk of perioperative morbidity and mortality. 


\section{References}

1. Writing Group Members, Mozaffarian D, Benjamin EJ, Go AS, Arnett DK, Blaha MJ, Cushman M, et al; American Heart Association Statistics Committee and Stroke Statistics Subcommittee. Heart disease and stroke statistics-2016 update: a report from the American Heart Association. Circulation. 2016;133; e38-360.
2. Cohn PF, Gorlin R, Cohn LH, Collins JJ Jr. Left ventricular ejection fraction as a prognostic guide in surgical treatment of coronary and valvular heart disease. Am J Cardiol. 1974;34:136-41.

3. Langer NB, Ando M, Simpson M, van Boxtel BS, Sorabella RA, Patel V, et al. Influence of left ventricular ejection fraction on morbidity and mortality after aortic root replacement. J Thorac Cardiovasc Surg. 2019;158:984-91.e1. 